\title{
COVID-19 INFECTION IN A CANDIDATE FOR AUTOLOGOUS HEMATOPOIETIC STEM CELL TRANSPLANTATION: A CASE REPORT
}

Mateus Lavor Lira; Yara Ceres e Silva Ferreira Lima; Isaias Lima de Figueiredo Júnior; Osvaldo Pimentel Oliveira Neto; Isabella Araújo Duarte; Beatrice Araújo Duarte; Fernando Barroso Duarte.

All authors from Walter Cantidio University Hospital, Federal University of Ceara

Correspondence to: yaraceres@hotmail.com

\begin{abstract}
The pandemic for the new coronavirus SARS-CoV-2 has been the causeof enormous challenges for the entire health system, especially in programs who dealwith Hematopoietic Stem Cell Transplantation (TCTH), since sequelae related to COVID-19 can be a hindrance to a possible HSCT. In case report, VBF, 61 years old, diagnosis of classic lymphocyte-rich Hodgkin'slymphoma in 2018 with initial treatment with ABVD, due to the return of the disease, an ICE regimen was started, but with disease progression after 5 cycles. Then, an IGEV scheme was started with a schedule of autologous hematopoietic cell transplantation, which took place in the third cycle in May / 2020. However, at the end of May / 2020, he was admitted to the emergency department with confirmation of SARS-Cov-2 infection by means of PCR of the nasal and oropharyngeal swab. He evolved during hospitalization with hypoxemic respiratory failure, mechanical ventilation and signs of secondary pulmonary infection, using multiple antimicrobial regimens, showing improvement and finally being extubated. However, he presented important pulmonary sequelae, with chest $\mathrm{CT}$ showing extensive cavitation in the left upper lobe and reticular opacities, with distortion of the pulmonary architecture. He was reassessed as to the possibility of autologous hematopoietic cell transplantation, but this was contraindicated due to pulmonary sequelae. In the case reported, the patient complied with the formal indication for HSCT, which would be refractoriness or relapse in a second remission in patients up to 70 years old with sensitivity to rescue schemes. However, due to pulmonary sequelae acquired after COVID-19, HSCT was contraindicated. This case leads us to the conclusion that the pandemic by the SARS-CoV-2 coronavirus can directly affect HSCT services and that in addition to preventing infection in this group of patients, they should be reevaluated after the recovery of COVID-19 for evaluation of structural and functional respiratory sequelae.
\end{abstract}

Keywords: Hematopoietic Stem Cell Transplantation, SARS-CoV-2

\section{INTRODUCTION}

The pandemic for the new coronavirus SARS-CoV-2 has been the cause of enormous challenges for the entire health system, especially in programs who deal with Hematopoietic Stem Cell Transplantation (HSCT).

Due to the lack of specific treatments and the high mortality of this infection in hematological patients[1], centers that deal with bone marrow transplantation have been paying special attention to the entire process, from a more rigorous assessment of donors, monitoring of recipients to testing the health team who watches them[2] .
However, care must also be directed to patients who are still in the process of indicating a transplant, since sequelae related to COVID-19 can be a hindrance to a possible HSCT.

HSCT has been shown to be one of the most effective treatments in patients with Hodgkin's or non-Hodgkin's Lymphoma who have relapses ${ }^{3}$, with the contraindication to the procedure being a poor prognostic factor for hematological disease.

In this work we report a case in which a patient with Hodgkin's lymphoma who had his Hematopoietic Stem cell transplant contraindicated due to sequelae related to COVID-19. 


\section{CASE REPORT}

VBF, 61 years old, diagnosis of classic lymphocyte-rich Hodgkin's lymphoma in 2018 with initial treatment with ABVD (Adriamycin + Bleomycin + Vinblastine + Dacarbazine) from december to july showing partial remission.

Subsequently, due to the return of the disease, an ICE regimen (Ifosfamide, Etoposide, Carboplastine) was started, but with disease progression after 5 cycles. Then, an IGEV scheme (ifosfamide, gemcitabine, vinorelbine) was started with a schedule of autologous hematopoietic cell transplantation, which took place in the third cycle in May / 2020.

At the end of May / 2020, he was admitted to the emergency department with productive cough, fever and dyspnea 4 days prior to admission, with confirmation of SARS-Cov-2 infection by means of PCR of the nasal and oropharyngeal swab. He evolved during hospitalization with hypoxemic respiratory failure and the need for intubation and mechanical ventilation, being admitted to the covid ICU of the Hospital Universitário Walter Cantídio on May 2020.

He showed signs of secondary pulmonary infection in the ICU, using multiple antimicrobial regimens, showing improvement and finally being extubated. However, he presented important pulmonary sequelae, with chest $C T$ showing extensive cavitation in the left upper lobe and reticular opacities, with distortion of the pulmonary architecture (Image 1).
After returning to the ward, no longer using antibiotics, he was reassessed as to the possibility of autologous hematopoietic cell transplantation, but this was contraindicated due to pulmonary sequelae.

Considering the contraindication to HSCT, the patient was discharged with the schedule of trying a new rescue chemotherapy scheme.

\section{DISCUSSION}

In the scientific society is known about the role of coronaviruses in serious infections that affect humans and animals. In December 2019, a new coronavirus was identified and confirmed as causing pneumonia in citizens of the city of Wuhan, China, which spread rapidly around the world. In February 2020, the World Health Organization confirmed that COVID-19, a disease that causes coronavirus, was then called SARS-CoV-2, coronavirus 2 for severe acute respiratory syndrome.

After contact with the virus, the disease developed in up to 14 days, with manifestations starting between four and five days more common. The most common symptoms are a common flu syndrome, with involvement of the upper respiratory tract, myalgia, low fever, diarrhea, asthenia, taste and smell disorders, the latter two being more common in COVID-19 in other viral infections. Pneumonia appears as the most common serious manifestation of the disease, manifested by dry cough, high fever,

Image 1 - Computed Tomogphy.
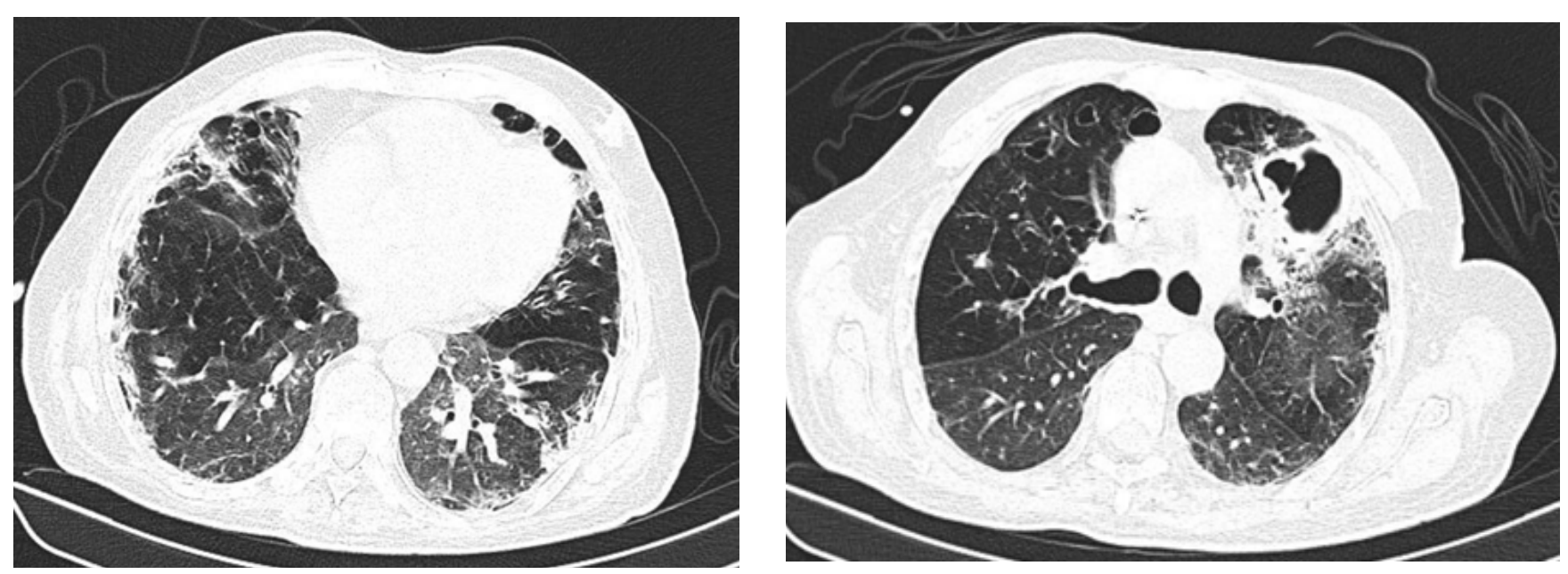
dyspnea associated with hypoxia and bilateral infiltrate seen on chest tomography, and also of variable spectrum, reaching severe respiratory failure, shock and organ dysfunction (injury acute kidney disease, myocarditis, liver damage).

Regarding the profile of patients with more severe disease, what was most observed was with regard to comorbidities. It was seen that in older individuals, over 60 years, with, mainly, cardiovascular disease, diabetes mellitus, obesity, hypertension, neoplasia (hematological, pulmonary and metastatic), chronic lung disease, smoking were associated with a more severe incidence of the disease and higher mortality.

Among the serious complications of the disease, the main one was respiratory failure due to Acute Respiratory Discomfort Syndrome (ARDS) associated with severe disease, with a good part of these patients being candidates for invasive ventilatory support. Cardiovascular complications were also observed, such as arrhythmias, acute cardiac injury (myocarditis) and shock. Thromboembolism in the form of a stroke in younger patients and without exuberant risk factors for this, in addition to pulmonary embolism. Intense inflammatory responses, characterized as an "inflammatory storm", have been said to be the main cause of all complications resulting from prolonged infection by coronavirus 2 , both as already described and in other neurological manifestations in the form of neuropathies. Such exuberant inflammatory condition was evaluated by laboratory alterations such as elevation of d-dimer, C-Reactive Protein, ferritin, being also used as markers of prognosis and evolution of the disease. Finally, in the case of a severe patient with significant inflammatory changes, secondary infections were also part of complications, mainly pulmonary, whether or not they were related to mechanical ventilation.

The recovery time can vary in about two weeks in milder conditions and from three to six weeks in more severe conditions, in addition to being related to age and previous comorbidities. Among the most common persistent symptoms were dyspnea, asthenia, joint pain and non-anginal chest pain. There is still no relevant data regarding long-term sequelae related to COVID-19, but the little that has been seen and compared with other coronaviruses has the potential for lasting respiratory impairment. This idea is due to the long-term exacerbated inflammatory state, asso- ciated with the necessary therapy, such as the use of glucocorticoids, prolonged intubation and greater use of neuromuscular and sedative blockers. It is also unknown about post-intensive care syndrome or sequelae of ARDS, being seen as pulmonary changes in patients after COVID-19 persistent abnormalities of lung function in mild and severe pneumonias, the most common of which are reduced diffusion and restriction capacity, especially in the severe ones, with patients undergoing intensive care being left out and there was no basic data to know the risk of developing loss of lung function and it is not known for how long this change persisted.

Although there are limited data on the impact of COVID-19 on transplant candidates and bone marrow transplant therapy donors and recipients, there is sufficient concern that COVID-19 may have a significant impact on post-transplant or post-therapy outcomes

Based on this concern, the American Society for Transplantation and Cellular Therapy (ASTCT) advises that if SARS-CoV-2 is detected in a respiratory sample, cell therapy and hematopoietic cell transplantation procedures should be postponed, including in patients with high-risk malignancies. The postponement should occur until the patient is asymptomatic and undergoes at least two consecutive negative CRP tests, with approximately one week between exams, if available [4].

Approximately $3 \%$ of patients undergoing autologous bone marrow transplantation have severe pulmonary complications requiring mechanical ventilation [5]. Therefore, assessment protocols for BMT candidates usually include lung function assessments in the expectation of decreasing the number of serious complications with therapy.

In the case reported, the patient complied with the formal indication for HSCT, which would be refractoriness or relapse in a second remission in patients up to 70 years old with sensitivity to rescue schemes. However, due to pulmonary sequelae acquired after COVID-19, HSCT was contraindicated and another therapeutic option was chosen.

This case leads us to the conclusion that the pandemic by the SARS-CoV-2 coronavirus can directly affect HSCT services and that in addition to preventing infection in this group of patients, they should be reevaluated after the recovery of COVID-19 for evaluation. structural and functional respiratory sequelae. 


\section{REFERENCES}

1. Malard, F., Genthon, A., Brissot, E. et al. COVID-19 outcomes in patients with hematologic disease. Bone Marrow Transplant (2020). https://doi. org/10.1038/s41409-020-0931-4

2. Ljungman, P., Mikulska, M., de la Camara, R. et al. The challenge of COVID-19 and hematopoietic cell transplantation; EBMT recommendations for management of hematopoietic cell transplant recipients, their donors, and patients undergoing CAR T-cell therapy. Bone Marrow Transplant (2020). https://doi.org/10.1038/s41409-020-0919-0

3. Lancet JE, Rapoport AP, Brasacchio R, et al. Autotransplantation for relapsed or refractory Hodgkin's disease: long term follow-up and analysis of prognostic factors. Bone Marrow Transplantation 1998v.22, p. 265-71.

4. American Society for Transplantation and Cellular Therapy (ASTCT), INTERIM GUIDELINES FOR COVID-19 MANAGEMENT IN HEMATOPOIETIC CELL TRANSPLANT AND CELLULAR THERAPY PATIENTS Version 1.3 April 16, 2020.
5. Ho VT, Weller E, Lee SJ, et al. Prognostic factors for early severe pulmonary complications after hematopoietic stem cell transplantation. Biol Blood Marrow Transplant 2001; v.7, p. DOI: 10.1053/ bbmt.2001.v7.pm11349809

6. Mclntosh K. Coronavirus Disease 2019 (COVID-19): Clinical features. In: Hirsch M S, ed. UpToDate. Waltham, Mass.: UpToDate, 2020. https://www.uptodate.com/contents/coronavirus-disease-2019-covid-19-clinical-features. Access at July 26, 2020.

7. Anesi G L. Coronavirus Disease 2019 (COVID-19): Critical care and airway management issues. In: Manaker S, ed. UpToDate. Waltham, Mass.: UpToDate, 2020. https://www.uptodate.com/ contents/coronavirus-disease-2019-covid-19critical-care-and-airway-management-issues. Access at July 26, 2020. 